\title{
Mapa sonoro de área central da cidade de São Paulo
}

\section{Noise mapping of a central area of São Paulo city}

\author{
Michalski, Ranny; Acamine, Érica² \\ ' Faculdade de Arquitetura e Urbanismo, Universidade de São Paulo, Rua do \\ Lago, 876, São Paulo, SP, 05508-080, Brasil, rannym@usp.br \\ 2 Faculdade de Arquitetura e Urbanismo, Universidade de São Paulo, \\ ericasaori@hotmail.com
}

\begin{abstract}
RESUMO
No município de São Paulo, a Lei 16.499, publicada em 2016, estipula um prazo de sete anos para o desenvolvimento e implantação do Mapa de Ruído Urbano da cidade. Diante da atual demanda, o trabalho consiste na simulação em modelo computacional do ruído ambiental urbano em uma fração da cidade de São Paulo, de forma a contribuir com as necessidades da mesma. Embora esta demanda esteja atrasada, se comparada aos países europeus, este é um importante passo no Brasil. A área urbana escolhida está localizada na Zona Central da cidade de São Paulo, no bairro da Consolação. A região possui vias de intenso tráfego e ruído: Avenida Dr. Arnaldo e Rua da Consolação, além da Avenida Paulista, com grande fluxo de automóveis ao longo do dia. O método de simulação utilizado na pesquisa é o previsional, apoiado pelo método experimental, com medições in situ. São apresentados os resultados parciais do estudo, como parte da pesquisa em desenvolvimento. Os resultados apontam que os níveis sonoros ultrapassam valores estabelecidos em normas técnicas e na legislação, sendo recomendada alguma ação para controle ou redução de ruído no local.
\end{abstract}

Palavras-chave: Mapeamento sonoro, Acústica ambiental, Acústica urbana, Planejamento urbano.

\begin{abstract}
In the city of São Paulo, the law 16.499, published in 2016, establishes a period of seven years for the development and implementation of the City Urban Noise Map. Given the current demand, the present research consists in the computer simulation of urban environmental noise at a fraction of the city of São Paulo, in order to contribute to the current needs of the city. Although this demand is late in comparison with European countries, this is one important step in Brazil. The chosen urban area is placed in the Central Zone of São Paulo, in Consolação neighborhood. The region is surrounded by high traffic and noise routes: Dr. Arnaldo Avenue, Consolação Street, and Paulista Avenue, with intense car flow throughout the day. The simulation method used in the research is the numerical, supported by the experimental method, with in situ measurements. Since the research is in development, partial results are presented. The results indicate that the sound levels surpass values established in technical standards and legislation, and some action is recommended to control or reduce local noise.
\end{abstract}

\footnotetext{
${ }^{1}$ MICHALSKI, Ranny; ACAMINE, Érica. Mapa sonoro de área central da cidade de São Paulo. In: II SIMPÓSIO
} NACIONAL DE GESTÃO E ENGENHARIA URBANA: SINGEURB, 2019, São Paulo. Anais... Porto Alegre: ANTAC, 2019. 
Keywords: Noise mapping, Environmental acoustics, Urban acoustics, Urban planning.

\section{INTRODUÇÃO}

A polvição sonora nas áreas urbanas é assunto de extrema importância, sendo considerada problema de saúde pública pela Organização Mundial da Saúde (WHO, 1999). Ruído devido a tráfego, vizinhos, máquinas, indústrias, atividades comerciais e recreativas incomoda e prejudica a qualidade de vida da população. Além das próprias fontes sonoras, a urbanização e o adensamento vertical nas cidades formam cânions urbanos que funcionam como caixas de ressonância acústica. A exposição prolongada a altos níveis de ruído pode causar danos à saúde, desde efeitos psicológicos à perda auditiva. Portanto, a qualidade acústica dos ambientes é fator crucial para o bem-estar e o conforto dos seus usuários.

No caso de espaços urbanos abertos, o tratamento acústico costuma ser complexo, pois muitas variáveis influenciam na qualidade acústica, além de uma grande variabilidade de condições tipológicas, microclimáticas e de usos. A análise do ruído nos centros urbanos é necessária e o desenvolvimento de modelos computacionais acompanha essa necessidade. Os softwares de simulação acústica de ruído urbano utilizam modelos capazes de analisar diversas variáveis e realizar cálculos simultâneos para análise e mapeamento sonoro.

A Organização Mundial da Saúde destaca ainda a importância do gerenciamento e controle de ruído em países em desenvolvimento. Este gerenciamento de ruído já é feito em países desenvolvidos há algumas décadas. A Diretiva Europeia de 2002 (PARLAMENTO EUROPEU, 2002), por exemplo, possui três partes principais: uma relativa ao mapeamento de ruído (obrigatório para cidades acima de 250 mil habitantes), um programa de ações e metas para combate à polvição sonora, e um sistema de informação ao público. No Brasil há apenas dois programas de mapeamento oficial, o de Fortaleza (BRITO e COELHO, 2013) e o de São Paulo (SÃO PAULO, 2016), mas existem iniciativas de pesquisas em algumas cidades (CORTÊS, 2018).

No município de São Paulo, a Lei 16.499, publicada em 2016, obriga a edição do Mapa de Ruído Urbano da cidade e estipula um prazo de sete anos para seu desenvolvimento e implantação (SÃO PAULO, 2016). Com o mapa de ruído será possível detectar áreas que apresentam níveis sonoros que ultrapassam limites permitidos pela legislação, e identificar as fontes emissoras para criar metas, planos de ação e prazos para reduzir ou controlar o ruído excessivo. Como consequência, a população se beneficiará com o processo.

Diante da atual demanda, o objetivo do presente trabalho consiste em simular um modelo computacional do ruído ambiental urbano em uma fração da cidade de São Paulo, mais especificamente, um trecho do bairro da Consolação. Embora esta demanda esteja atrasada em relação aos países europeus, este é um importante passo no Brasil. A pesquisa contribui também para disseminar a utilização de modelagem computacional em acústica urbana na Faculdade de Arquitetura e Urbanismo da Universidade de São Paulo.

\section{MAPA SONORO}

A principal ferramenta para o diagnóstico da distribuição e quantificação do ruído em áreas determinadas são os mapas sonoros, também chamados de mapas de ruído. O mapa sonoro de uma área geográfica fornece informação visual do comportamento acústico de tal área, num determinado momento. Pode-se dizer que o mapa sonoro fornece uma maneira de ver o que é invisível (visto que assim são os níveis sonoros).

Os mapas sonoros podem ter muitas utilidades, como, por exemplo: identificar onde e em qual intensidade estão as pessoas expostas a níveis excessivos de ruído, determinar o número de pessoas e de habitações em uma zona específica expostas a determinados níveis de ruído, auxiliar no projeto de soluções mitigadoras do problema, além de servir como ferramenta de apoio a decisões de planejamento e ordenamento urbano com relação ao gerenciamento e controle de ruído nas cidades.

Os mapas podem ser elaborados a partir de medições, modelos matemáticos preditivos ou uma combinação de ambos. Alguns softwares específicos estão disponíveis no mercado, 
como o CadnaA (DataKustik), o SoundPLAN (SoundPLAN GmbH) e o LimAPredictor (Brüel \& Kjaer). Para a construção do modelo acústico, são necessários os dados de entrada. Por fim, recomendam-se medições acústicas em pontos estratégicos para validação do modelo (EUROPEAN COMISSION, 2006; GONZÁLEZ, 2016).

\section{DESENVOLVIMENTO}

A seguir são descritas as etapas para elaboração do mapa sonoro.

\subsection{Trecho selecionado}

A área selecionada foi um trecho do bairro da Consolação, ligando a Faculdade de Saúde Pública da USP ao Centro Universitário Maria Antônia, na zona central da cidade de São Paulo. As Figuras 1 e 2 ilustram o local. O bairro é composto principalmente por edifícios residenciais, escolares e comerciais. A região é cercada por quatro vias: Avenida Dr. Arnaldo, Rua da Consolação, Avenida Angélica e Rua Maria Antônia (que vira Avenida Higienópolis). As vias de intenso tráfego e ruído ao longo do dia são a Avenida Dr. Arnaldo e a Rua da Consolação, além da Avenida Paulista.

Figuras 1 e 2 - Inserção urbana e vista aérea do trecho selecionado.

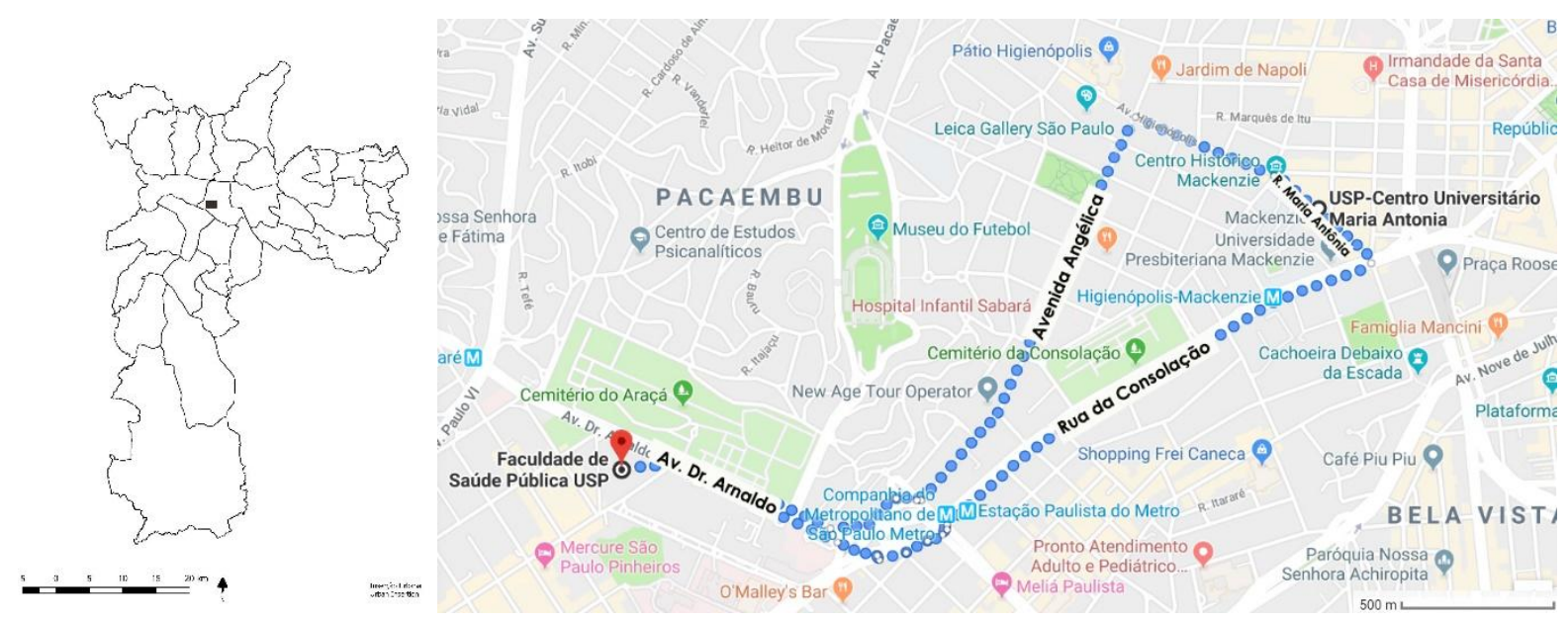

Fontes: GeoSampa e Google Maps.

\subsection{Metodologia}

As atividades da pesquisa estão fundamentadas em dois procedimentos distintos, mas complementares: um método do tipo dedutivo numérico ou previsional (simulações computacionais de modelos preditivos), apoiado por outro método do tipo indutivo experimental (levantamentos em campo).

A primeira etapa da pesquisa é o levantamento de informações sobre a área em estudo, incluindo informações técnicas e desenhos, reunindo plantas, cortes, elevações, orientação e detalhes. A segunda etapa corresponde à modelagem computacional do local. A terceira etapa corresponde à inserção dos dados de entrada no programa e realização das simulações. Os dados de entrada são necessários para a construção do modelo acústico e são determinantes no resultado final obtido. A quarta etapa é a validação do modelo, através da comparação dos resultados das simulações com os resultados das medições acústicas.

Após a seleção do trecho a ser estudado e o levantamento de informações sobre o local, foi realizada sua modelagem computacional. A base utilizada para caracterizar a geometria da área, incluindo topografia, edificações e vias, foi obtida a partir do mapa digital da cidade de São Paulo, disponível no site da plataforma pública GeoSampa.

O software utilizado para as simulações é o CadnaA (Computer Aided Noise Abatement), um 
programa de predição acústica capaz de calcular, avaliar, predizer e apresentar a exposição de ruído e o impacto sonoro em ambiente externo. No programa, todas as fontes de ruído são inseridas e todos os fatores específicos necessários são configurados. Após a simulação, os resultados das medições em campo e no modelo computacional devem ser comparados, para validar a simulação.

O CadnaA possui um módulo de importação de arquivos do tipo ESRI shapefile, que contém informações geográficas (formas vetoriais), atributos no formato dBase (tabela de atributos) e índice que posiciona a geometria. O modelo utilizado no trabalho é desse tipo.

O passo seguinte foi a inserção dos dados de entrada no software: tipos de vias e asfalto, número de veículos por hora, porcentagem de veículos pesados, limites de velocidade. A principal fonte de ruído é o tráfego rodoviário, sendo utilizada a metodologia de cálculo alemã RLS 90.

Dados de entrada foram obtidos no Relatório "Pesquisa de monitoração da mobilidade no sistema viário principal: Volume e Velocidade - 2017", disponível no site da Companhia de Engenharia de Tráfego (CET, 2017). Para a Rua da Consolação, os valores foram: 2811 veículos leves por hora, incluindo automóveis e motocicletas, e 148 veículos pesados por hora. Para as ruas locais, de pequeno porte, foram realizadas contagens de veículos em campo, no período da manhã do dia 26 de abril de 2019, Figuras 3 e 4, obtendo uma média de 300 veículos leves por hora e 1 veículo pesado por hora.

O mapa sonoro simulado refere-se a uma altura de $1,7 \mathrm{~m}$ e período diurno. Na área apresentada na Figura 2, foram escolhidos doze pontos de medição, entretanto, apenas o Ponto 1 se encontra no trecho utilizado na fase inicial de simulação. O ponto de medição Pl, indicado na Figura 5, foi escolhido devido à fonte sonora ser predominantemente de um único tipo, a Rua da Consolação, com maior circulação de veículos (CORTÊS, 2018).

Para uma primeira validação do modelo, foi realizada uma comparação do resultado da simulação com o resultado de medições realizadas in situ no ponto Pl. A realização das medições acústicas seguiu as diretrizes estabelecidas pela norma NBR 10151 (ABNT, 2019). O sonômetro foi colocado a uma altura aproximada de $1,70 \mathrm{~m}$ do nível da rua e foi calculada a média energética de três medições de níveis de pressão sonora equivalentes ponderados em A, realizadas simultaneamente à contagem de veículos.

Figuras 3 e 4 - Contagem de veículos e medições em campo.
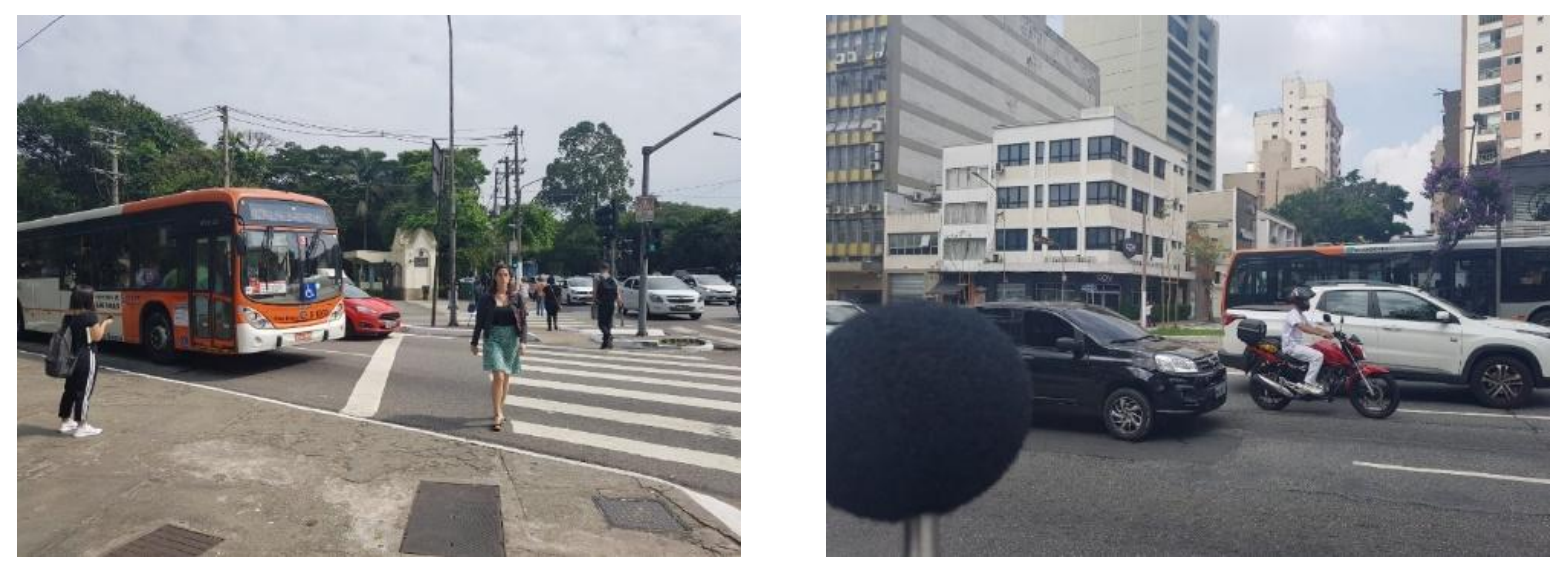

Fonte: Os autores

\section{RESULTADOS PARCIAIS}

O mapa sonoro, simulado na fase inicial para um trecho da Rua da Consolação, é apresentado na Figura 5, com a legenda das cores relativas aos níveis de pressão sonora, expressos em dB. É possível localizar no mapa regiões menos e mais ruidosas (próximas às vias de maior fluxo de veículos). A Tabela 1 apresenta o valor medido no ponto de medição 1 , juntamente com o valor simulado pelo CadnaA para o mesmo ponto. 
Figura 5 - Mapa sonoro parcial de trecho da Rua da Consolação.

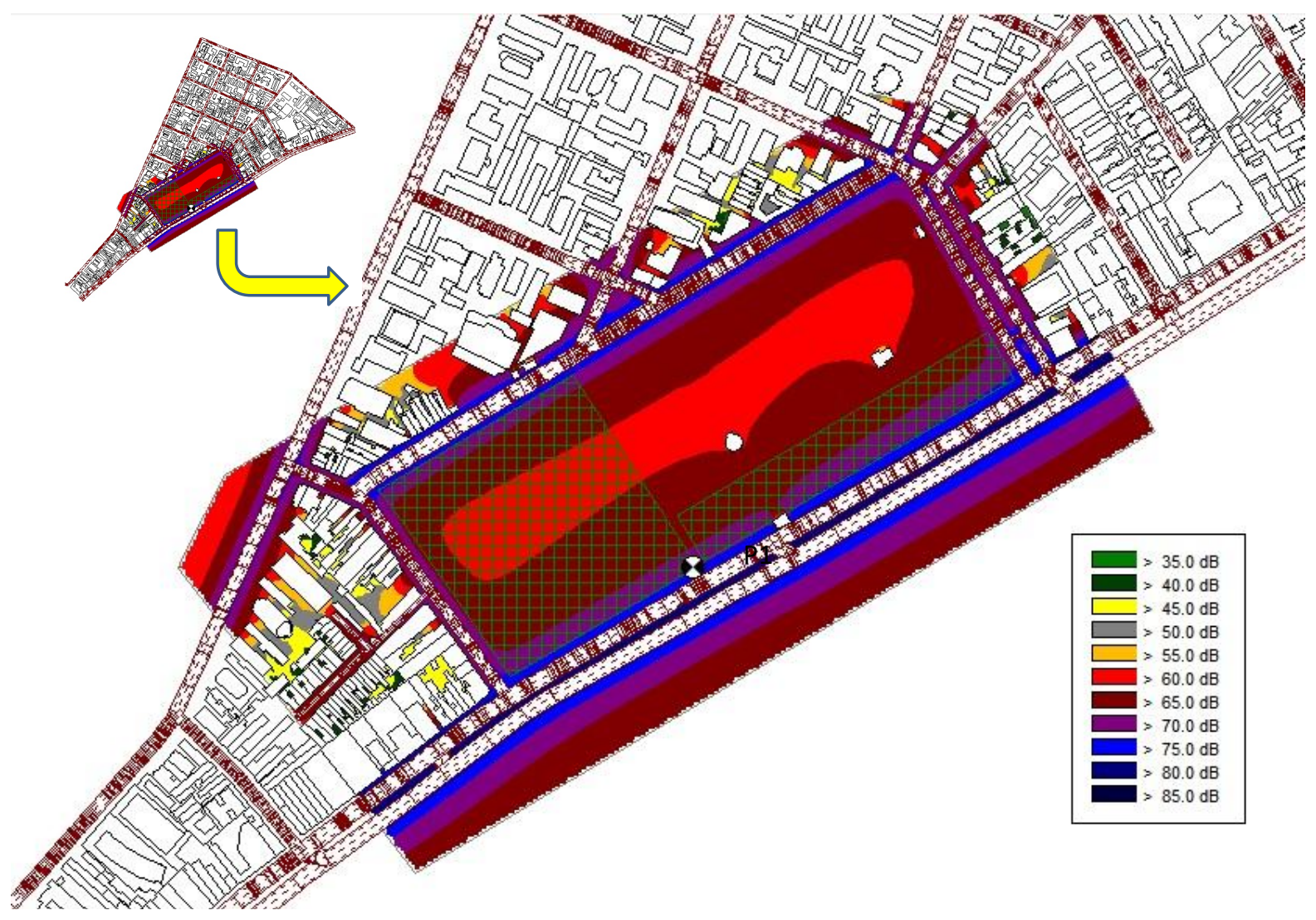

Fonte: Os autores

Tabela 1 - Níveis sonoros simulados e medidos.

\begin{tabular}{cccc}
\hline \multirow{2}{*}{ Pontos de medição/recepção } & \multicolumn{3}{c}{ LAeq (dB) } \\
\cline { 2 - 4 } P1- R. da Consolação X R. Pedro Taques & 74,0 & 73,0 & 1,0 \\
\cline { 2 - 4 }
\end{tabular}

Fonte: Os autores.

Os resultados obtidos na primeira etapa do estudo mostraram-se similares, de modo que a medição em campo demonstrou a eficácia e a acuidade do software. A etapa seguinte será a extensão da simulação para uma maior área do bairro, seguida por mais medições em campo de maneira a validar as simulações realizadas.

\section{CONCLUSÕES}

O comportamento acústico em espaços urbanos abertos pode ser avaliado por meio de simulações computacionais e apoiado por levantamentos empíricos in situ, para assim se obter uma análise comparativa com validação dos resultados.

Após análise dos resultados, é possível constatar que os níveis de ruído ultrapassam valores de normas e legislação. Por exemplo, o limite diurno de $60 \mathrm{~dB}$ estabelecido na versão revisada da norma NBR 10151 (ABNT, 2019) para área mista com predominância de atividades comerciais e/ou administrativas, é em muito ultrapassado. No caso da legislação municipal, o limite máximo estabelecido para o período diurno, de acordo com a Lei de Uso e Ocupação do Solo (SÃO PAULO, 2016), para o zoneamento do local (Zona Eixo de Estruturação da Transformação Urbana, ZEU) é de 60 dB, sendo também ultrapassado pelos níveis simulado e 
medido no ponto de medição. Mostra-se, portanto, necessário realizar alguma ação de planejamento para reduzir ou controlar o ruído no local, de forma a melhor a qualidade sonora no mesmo.

O trabalho encontra-se em desenvolvimento e fornece resultados parciais de mapeamento sonoro em trecho urbano na Zona Central de São Paulo. Os resultados apresentados mostram uma modelagem adequada do ambiente em estudo bem como a eficácia do software. Os passos seguintes correspondem à extensão da simulação para uma maior área.

\section{AGRADECIMENTOS}

Os autores agradecem ao Programa Unificado de Bolsas de Estudos para Apoio e Formação de Estudantes de Graduação da Universidade de São Paulo.

\section{REFERÊNCIAS}

ABNT ASSOCIAÇÃO BRASILEIRA DE NORMAS TÉCNICAS. NBR 10151: Acústica-Medição e avaliação de níveis de pressão sonora em áreas habitadas, Aplicação de uso geral. Rio de Janeiro, 2019.

BRITO, F; COELHO, B. The creation of the noise map of the city of Fortaleza. In: NOISE CONTROL ENGINEERING. INTERNATIONAL CONGRESS AND EXPOSITION. 42nd 2013, INTER-NOISE 2013, Institute of Noise Control Engineering, Innsbruck, Proceedings..., 2013.

CADNAA Software. Datakustik. Disponível em: <https://www.datakustik.com/products/ cadnaa/cadnaa/>. Acesso em: 20 abr. 2019.

CET COMPANHIA DE ENGENHARIA DE TRÁFEGO. Pesquisa de monitoração da mobilidade no sistema viário principal: Volume e Velocidade - 2017. Disponível em: <http://www.cetsp. com.br/media/714822/msvp-2017-volume-e-velocidade.pdf> Acesso em: 20 abr. 2019.

CORTÊS, M. M. Método de avaliação sonora em áreas urbanas formais e informais. Tese (doutorado) - UFRJ/ Faculdade de Arquitetura e Urbanismo / Programa de Pós-graduação em Arquitetura, Rio de Janeiro, 2018.

EUROPEAN COMISSION. Working Group Assessment of Exposure to Noise (WG-AEN). Good Practice Guide for Strategic Noise Mapping and the Production of Associated Data on Noise Exposure, version 2, 2006.

GONZÁLEZ, D. M. et al., ISO 1996 measurement procedure and the uncertainty associated in strategic noise maps. In: 22nd INTERNATIONAL CONGRESS ON ACOUSTICS, 2016, Buenos Aires, Proceedings..., 2016.

PARLAMENTO EUROPEU, DIRECTIVA 2002/49/CE DO PARLAMENTO EUROPEU E DO CONSELHO relativa à avaliação e gestão do ruído ambiente, 25 jun. 2002.

SÃO PAULO (Município). Lei n 16.499, de 20 de julho de 2016. Dispõe sobre a elaboração do Mapa do Ruído Urbano da Cidade de São Paulo e dá outras providências. Diário Oficial do Município de São Paulo. São Paulo, SP, 20 jul. 2016.

. Lei $n^{\circ} 16.402$, de 22 de março de 2016. Disciplina o parcelamento, o uso e a ocupação do solo no Município de São Paulo, de acordo com a Lei n 16.050, de 31 de julho de 2014 - Plano Diretor Estratégico (PDE). Lei de Parcelamento, Uso e Ocupação do Solo de São Paulo. Diário Oficial do Município de São Paulo. São Paulo, SP, 22 mar. 2016.

WHO WORLD HEALTH ORGANIZATION. Guidelines for Community Noise, 1999. Disponível em: <http://whqlibdoc.who.int/hq/1999/a68672.pdf>. Acesso em: 20 jul. 2018. 
GeoSampa, Mapa Digital da Cidade de São Paulo. Disponível em:

<http://geosampa.prefeitura.sp.gov.br/PaginasPublicas/_SBC.aspx>. Acesso em 20 dez 2018. 